Itinéraires Itinéraires

Littérature, textes, cultures

\title{
La modernité en question(s)
}

Jane Avner

\section{(2) OpenEdition}

\section{Journals}

Édition électronique

URL : http://journals.openedition.org/itineraires/1690

DOI : $10.4000 /$ itineraires. 1690

ISSN : 2427-920X

Éditeur

Pléiade

Édition imprimée

Date de publication : 1 décembre 2010

Pagination : 7-23

ISBN : 978-2-296-13183-5

ISSN : 2100-1340

\section{Référence électronique}

Jane Avner, «La modernité en question(s) », Itinéraires [En ligne], 2010-4 | 2010, mis en ligne le 01 décembre 2010, consulté le 22 septembre 2020. URL : http://journals.openedition.org/itineraires/1690 ; DOI : https://doi.org/10.4000/itineraires.1690

\section{$\Theta \oplus \Theta \Theta$}

Itinéraires est mis à disposition selon les termes de la licence Creative Commons Attribution - Pas d'Utilisation Commerciale - Pas de Modification 4.0 International. 


\title{
La modernité en question(s)*
}

\begin{abstract}
Things that are modern do not just sally forth in advance of their time. They also recall things forgotten; they control anachronistic reserves which have been left behind and which have not been exhausted by the rationality of eternal sameness.
\end{abstract}

Theodor Adorno, Quasi una fantasia, note 17.

\section{Introduction}

La modernité de Shakespeare n'est plus à démontrer; la modernité shakespearienne se démontre toujours. Depuis le célèbre hommage de Ben Jonson pour qui William Shakespeare «n'appartient à aucune époque car il est intemporel », jusqu'aux propos des adeptes d'un early modern Shakespeare, en passant, entre autres, par l'analyse de Jan Kott (Shakespeare, notre contemporain), la modernité shakespearienne n'a jamais cessé d'être soulignée et célébrée ${ }^{1}$. À partir des années 1980 et de la multiplication des études théoriques, particulièrement marquées dans les études renaissantes, notre curieuse tendance à façonner la modernité shakespearienne à notre propre image a souvent été soulignée avec humour par la critique. Tantôt Shakespeare serait «un lacanien sophistiqué » (Betteridge), tantôt il serait difficile de le lire «sans penser qu'il était familier avec l'œuvre de Hegel, de Marx, de Nietzsche, de Freud, de Wittgenstein, de Derrida ${ }^{2} \ldots$ » (Eagleton). Joel Fineman résume ainsi cet

\footnotetext{
* Je tiens à remercier Élise Avner et Gilbert Pham-Thanh pour leur aide précieuse dans la relecture de ce texte.

1. Ben Jonson, « He was not of an age, but for all time! », To the Memory of my Beloved, the Author Mr William Shakespeare : And what he hath left us, Poème dédicatoire, First Folio, 1623; Jan Kott, Shakespeare Our Contemporary, London, Methuen, 1965 [Pologne, 1964]. Le terme early modern n'a pas d'expression analogue satisfaisante en français, les différentes phases des XVI et $\mathrm{XVII}^{\mathrm{e}}$ siècles étant désignées par les termes Renaissance, Réforme, Baroque, et époque classique. Nous proposons donc de garder le terme si largement employé dans la critique anglo-saxonne.

2. Thomas Betteridge, Shakespearean Fantasy \& Politics, Hatfield, University of Hertfordshire Press, 2005, p. 42; Terry Eagleton, William Shakespeare, Oxford, Basil Blackwell, 1986,
} 
effet de miroir : « Soit Shakespeare était particulièrement avisé sur le plan théorique, soit la théorie est elle-même shakespearienne ${ }^{3}$. »

Lorsque l'expression early modern se répand dans la critique anglosaxonne pour désigner les $\mathrm{XVI}^{\mathrm{e}}$ et $\mathrm{XVII}{ }^{\mathrm{e}}$ siècles, la modernité devient un thème privilégié de la recherche shakespearienne car le rôle singulièrement créatif joué par Shakespeare dans le développement de la langue anglaise est, chacun le sait, déterminant. Sans la publication en 1623 du First Folio, « livre [...] que l'on pourrait surnommer le manuel de langue de la culture moderne ", les études d'une période dite early modern n'auraient sans doute pas vu le jour en Angleterre ${ }^{4}$. Au moment où la langue anglaise se modernise et prend son essor, et alors que les tout premiers théâtres s'établissent à Londres, l'envergure, l'éclat et l'originalité de son œuvre placent donc Shakespeare au cœur des débats concernant la modernité. Lorsqu'il s'approprie des textes mythiques, légendaires, poétiques ou historiques, Shakespeare pratique ce que Harold Bloom appelle «strong misreadings » ou « poetic misprision $^{5} »$. Que peuvent nous dire de la modernité shakespearienne ces relectures décalées, ces « méprises » poétiques volontaires? Et d'autre part, quel genre d'échanges s'effectue dans les réécritures auxquelles l'œuvre shakespearienne n'a jamais cessé de donner lieu?

En ce début du $\mathrm{XXI}^{\mathrm{e}}$ siècle, que la critique n'hésite pas à appeler " post-théorique », le présent recueil propose de reprendre le problème de la modernité shakespearienne, singulière, voire plurielle, à partir des multiples approches théoriques des dernières décennies. Partant de la tension féconde qu'implique l'expression early modern, nous nous proposons de placer cet ouvrage sous le signe de Janus, d'une part en poursuivant la réflexion engagée par la critique sur la manière dont Shakespeare lui-

p. ix-x. Richard Wilson note que la plaisanterie de Eagleton avait été anticipée par Daniel Mesguich dans «The Book to Come is the Theatre », Sub-Stance, $\mathrm{n}^{\circ}$ 18/19, 1977, p. 118. Voir Richard Wilson, Shakespeare in French Theory : King of Shadows, Londres, Routledge, 2007, p. 261. Prenant ces plaisanteries au pied de la lettre, Richard Wilson s'est penché sur les relations qui se sont tissées entre l'œuvre shakespearienne et ce que la critique anglo-saxonne appelle « French Theory ». Dans cette étude l'auteur explore les affinités électives, l'étrange jeu d'ombres qui s'est joué entre Shakespeare et les intellectuels français des dernières décennies. Il s'agit non seulement de lire Shakespeare à la lumière de la critique littéraire et surtout philosophique française, mais aussi, ce qui est moins habituel, de lire la Théorie française à l'ombre de Shakespeare, "whose shadow shadows doth make bright» (« dont l'ombre donne aux ombres la clarté » (sonnet 43), trad. française de Jean Fuzier, Euvres Complètes de Shakespeare, vol. 1, Paris, Gallimard, coll. « Bibliothèque de la Pléiade », 1959.

3. Joel Fineman, The Subjectivity Effect in Western Literary Tradition, Cambridge, MA, MIT Press, 1991, p. 112.

4. Douglas Bruster, « Shakespeare and the End of History : Period as Brand Name », dans Hugh Grady (ed.), Shakespeare \& Modernity-Early Modern to Millenium, London \& NY, Routledge, 2000, p. 186.

5. Harold Bloom, The Anxiety of Influence : A Theory of Poetry [1973], Oxford, Oxford University Press, 1997, p. xxiii. 
même s'inscrivait dans la modernité; d'autre part en nous penchant sur la thématique de la traduction, de la mise en scène, ainsi que des réécritures en tout genre (romanesque, théâtrale, filmique), qui explorent, de façons très diverses, la modernité shakespearienne. La présentation qui suit tente de dégager, de manière nécessairement succincte et subjective, quelques aspects clés de la problématique de la modernité shakespearienne.

\section{Le temps en question}

L'expression early modern, si largement répandue dans la critique littéraire anglo-saxonne, s'est effectivement avérée "féconde ", créant de nouvelles perspectives pour d'autres types d'histoire, ou des histoires " autres », que celles que désigne le terme « Renaissance ${ }^{6} »$. Cependant elle n'est pas sans soulever de nombreuses questions. Comment faut-il entendre des termes qui, comme le note Terence Cave, sont déictiques - comme l'adverbe «maintenant »- mais qui désignent en même temps une période historique révolue ${ }^{7}$ ? Autrement dit, comment articuler le couple antinomique que forment les deux éléments de cette expression? Quand commence la modernité? Et question inhérente à celle-ci : qu'estce que la modernité? Vaste champ d'interrogations dont évidemment nul ne peut faire le tour, les contours du passé changeant, comme chacun sait, au fil des questions qu'on lui pose.

Si l'expression early modern n'est pas récente, c'est surtout à partir des années 1950 qu'elle commence à figurer de plus en plus fréquemment dans les titres de livres d'histoire sur les $\mathrm{XVI}^{\mathrm{e}}$ et XVII $\mathrm{X}^{\mathrm{e}}$ siècles, puis apparaît dans la très influente revue d'histoire Past and Present, où elle est employée par d'éminents historiens de la période tels que Lawrence Stone ${ }^{8}$. Son adoption par la critique littéraire, notamment celle d'inspiration marxiste - le nouvel historicisme et le matérialisme culturel - a très sensiblement marqué les études shakespeariennes. En effet, à partir de la fin des années 1970, la critique littéraire privilégie une approche plus diachronique de l'œuvre shakespearienne, en opposition à l'approche anhistorique et synchronique de la théorie de la Nouvelle Critique des décennies 1950 et 1960. Problématique à plusieurs égards, la migration de l'expression

6. Cependant le terme « Renaissance » n'a pas été complètement abandonné pour autant. Pour un excellent résumé et une discussion de ce problème voir Terence Cave, «Locating the Early Modern », Paragraph, vol. 29, n 1, mars 2006, p. 12-26; Douglas Bruster, op. cit.; David Aers, "A Whisper in the ears of Early Modernists; or, Reflections on Literary Critics Writing the "History of the Subject" ", dans David Aers (ed.), Culture and History 1350-1600 : Essays on English Communities, Identities and Writing, Detroit, Wayne State University Press, 1992, p. 172-202 et particulièrement p. 194-196.

7. Terence Cave, op. cit., p. 14.

8. Parmi d'autres études, George Clark, Early Modern Europe : from about 1450-1720, London, Oxford University Press, 1957; voir également Lawrence Stone, « Social Mobility in England : 1500-1700», Past \& Present, vol. 33, n 1, 1966, p. 38-59. 
early modern d'une discipline (l'histoire) à une autre (la littérature), permet néanmoins de repenser la thématique de la modernité : désormais c'est le caractère opportun (timeliness) plutôt que l'intemporalité (timelessness) de l'œuvre shakespearienne qui, n'en déplaise à Ben Jonson, est interrogé et analysé. Et bien que tous les personnages shakespeariens se distinguent par leur complexité psychologique de ceux du théâtre Tudor et médiéval, c'est Hamlet, pour qui le temps est si notoirement « disloqué », qui serait presque universellement reconnu comme figure emblématique d'une subjectivité moderne. Au moment où le siècle touche à sa fin, c'est à travers ses nombreux soliloques que ce héros inquiet, hanté par un passé qui refuse justement de passer, et par un avenir incertain, exprime ce que Joel Fineman appelle une "nouvelle subjectivité poétique ${ }^{9} »$. Shakespeare joue un rôle central dans le façonnement de «l'individu nouveau, celui qui découvre une conscience de soi qui est aussi conscience tragique du monde », écrit Line Cottegnies ${ }^{10}$.

La notion d'intériorité qui caractérise les nouvelles formes de subjectivité est controversée. La recherche sur l'histoire de la construction du sujet supposé « moderne » est fertile et la grande divergence de points de vue témoigne de la complexité de ce problème sur lequel, comme l'écrit Muriel Cunin dans ce volume, aucun consensus ne s'est dégagé ${ }^{11}$. Pour certains, la notion d'intériorité communément associée à Hamlet est anachronique, simple prémisse d'une "subjectivité bourgeoise » qui, elle, n'apparaîtra qu'au XVIII ${ }^{\mathrm{e}}$ siècle ${ }^{12}$. Cette subjectivité moderne en devenir que les critiques tels que Francis Barker reconnaissent chez Hamlet ne s'exprimerait, selon eux, qu'au début de la pièce et se limiterait curieusement aux assurances que le Prince donne à sa mère dans la deuxième scène, concernant la valeur des signes visibles de son chagrin. Il ne connaît pas, dit-il, « le semble». Aucun des signes extérieurs - « les habits coutumiers d'un noir solennel», « les soupirs haletants », « la mine accablée », bref « aucune des formes, modes ou figures du chagrin » ne peuvent «peindre » Hamlet « au vif» car en effet « elles semblent». Il s'agit « des actions qu'un homme peut jouer », mais il a en lui, dit-il « ce qui passe le paraître ${ }^{13} »$.

9. Joel Fineman, Shakespeare's Perjured Eye: The Invention of Poetic Subjectivity in the Sonnets, Berkeley, University of California Press, 1986, p. 46.

10. «Introduction », Théâtre Élisabéthain, vol. 2, Paris, Gallimard, coll. « Bibliothèque de la Pléiade », 2009, p. xxxi.

11. Voir «"Still keep/My bosom franchised" : Macbeth et l'espace intérieur » dans ce volume.

12. Francis Barker, The Tremulous Private Body, New York, Methuen, 1984, p. 31-58; Catherine Belsey, The Subject of Tragedy: Identity and Sexual Difference in Renaissance Drama, New York \& London, Methuen, 1985, p. 48; Jean Howard, « The New Historicism in Renaissance Studies ", English Literary Renaissance, n ${ }^{\circ}$ 16, 1986, p. 15.

13. I.ii.69-79, trad. de Jean-Michel Déprats, Granit, s. 1., 1986. 
Le point de vue de Barker, assez largement partagé par la critique 《 néo-historiciste » a été vivement contesté quelques années plus tard, notamment par le médiéviste David Aers, dans un article qui a fait date (1992) ${ }^{14}$, ainsi que par Katharine Eisaman Maus dont la remarquable étude publiée en 1995 établit une distinction entre les concepts d'intériorité (inwardness), et de subjectivité ${ }^{15}$. Bien que leurs réponses, fouillées, denses, représentent des approches très différentes, tous deux pointent une des difficultés qui guette l'appropriation du terme early modern par la critique littéraire. Comme l'implique le titre malicieux de la réponse qu'Aers adresse aux « critiques littéraires en train d'écrire "l'histoire du sujet" ", l'historicité de cette "histoire» pose problème : celle écrite par Barker et al. fait du Moyen Âge une période homogène, mythique, idéalisée. Dénonçant une vision qui ne tient compte ni des textes littéraires, religieux ou philosophiques, ni de la recherche récente, largement inspirée, comme celle des $\mathrm{XVI}^{\mathrm{e}}$ et $\mathrm{XVII}^{\mathrm{e}}$ siècles, par les nouvelles méthodologies théoriques, Aers reproche aux early modernists d'ignorer la complexité et la diversité des expressions d'intériorité qui caractérisent la période médiévale. Eisaman Maus, quant à elle, démontre que la méfiance d'Hamlet - son sens aigu d'un moi intérieur - qui ne pourrait ou ne devrait pas être révélé, est très développée dans l'Angleterre du $\mathrm{XVI}^{\mathrm{e}}$ siècle. Ce sont, ditelle, les enjeux des afflictions et les satisfactions attenantes à la différence entre une intériorité inexprimée et une extériorité théâtralisée, ainsi que les inquiétudes épistémologiques qu'un tel hiatus génère, qu'explore le théâtre de Shakespeare, de Marlowe et de Jonson ${ }^{16}$.

Si l'on peut aisément reconnaître avec Aers que la périodisation de l'histoire pose un problème, il est néanmoins clair que les XVI et XVII ${ }^{e}$ siècles voient l'émergence de nouvelles formes de subjectivité, que l'œuvre shakespearienne configure de manière saisissante. Fineman a raison de parler de l'invention d'une nouvelle "subjectivité poétique » dans les Sonnets, et Cottegnies d'observer que l'on a pu décrire Hamlet comme «l'une des premières tragédies de la subjectivité moderne, où la découverte de l'intériorité passe par des formes d'introspection inédites au théâtre ${ }^{17} »$. Pour Anthony Burgess, Hamlet, produite en 1599, "résume toute l'ère élisabéthaine qui s'achève, ère écartelée entre un héritage médiéval et un scepticisme

14. David Aers, op. cit., p. 172-202.

15. Katharine Eisaman Maus, Inwardness and Theater in the English Renaissance, Chicago, University of Chicago Press, 1995. Pour la distinction entre inwardness et subjectivity, voir p. 30 .

16. Ibid., p. 2. Voir la discussion de cette thèse par Muriel Cunin ainsi que l'étude de Lacey Baldwin Smith, qui analyse le climat de peur et de suspicion qui régnait dans l'Angleterre du XVI ${ }^{\mathrm{e}}$ siècle (Treason in Tudor England: Politics and Paranoia, London, Jonathan Cape, 1986).

17. Line Cottegnies, op. cit., p. xxxi. 
nouveau ${ }^{18} »$. Les deux sensibilités cohabitent en la personne du Prince, et cette indétermination existentielle est, pour nous, l'un des aspects clés de l'esthétique novatrice, et résolument moderne, de Shakespeare. Car il n'est pas rare, comme l'observe Howard Felperin, que Shakespeare affuble des personnages de tous les accoutrements d'une théâtralité archaïque afin de les identifier à un ordre vieillissant. Il agit en médiateur avisé du changement théâtral, concentrant les éléments archaïques en certains points stratégiques de ces pièces afin de les problématiser : il les invalide au moment même où il les inclut. À travers Le Meurtre de Gonzague, le centre archaïque d'Hamlet, nous regardons « la pièce de Shakespeare approcher et, pour ainsi dire, embrasser son propre prototype avant de se retourner et de la fuir, d'esquisser une espèce de chorégraphie faite de rencontres et de séparations ${ }^{19} »$.

Shakespeare partage avec d'autres dramaturges de l'époque un goût pour le trope des pièces enchâssées, dont Le Meurtre de Gonzague est sans doute la plus célèbre. D'une surprenante diversité ces scènes en miroir ouvrent un espace réfléchissant ou méta-théâtral, formant à partir des toutes premières pièces un élément essentiel de la poétique shakespearienne. Déjà dans sa première tragédie de vengeance, Titus Andronicus, le mimétisme, principe même de la vengeance problématisé tout au long de la pièce, est lui-même mimé, mis en scène dans le masque du « Vengeur, Meurtrier et Violeur », joué devant Titus. La réflexivité explorée si magistralement dans cette scène signe dès le début de la carrière de Shakespeare une rupture décisive avec la tradition théâtrale antérieure. Titus Andronicus, si longtemps déconsidérée par nous "modernes", si profondément appréciée par le public originel, illustre plusieurs aspects de la modernité shakespearienne, et tout d'abord l'infaillible instinct d'homme de théâtre de son auteur. Titus Andronicus est la réplique - drôle, intelligente, ironique - des Comédiens du Chambellan à l'immense succès remporté par The Spanish Tragedy de Kyd. Si le public veut voir des tragédies de vengeance, alors il faut lui en donner. With a vengeance! En multipliant les horreurs, Shakespeare propose un drame jouissif, fine parodie du genre qui est en même temps une réflexion spirituelle sur les limites même du langage, sur le dicible et l'indicible. En homme kaïrique par excellence, Shakespeare a su guetter le moment opportun et faire de l'Occasio l'événement car « pour le génie créateur », comme l'écrit Vladimir Jankélévitch, «au lieu d'être une occurrence muette [la rencontre] devient une occasion riche de sens ${ }^{20} »$.

18. Anthony Burgess, English Literature, Londres, Longman, 1958, p. 81.

19. "We watch Shakespeare's play approach and embrace, as it were, its own archaic prototype, only to turn and flee it in an almost choreographic pattern of meeting and parting. » Howard Felperin, Shakespearean Representation: Mimesis \& Modernity in Elizabethan Tragedy, Princeton, New Jersey, Princeton University Press, 1977, p. 59-60.

20. Vladimir Jankélévitch, Quelque part dans l'inachevé, Paris, Gallimard, coll. «Folio Essais », 1978, p. 42. 


\section{La Théorie en question}

" L'âge d'or de la théorie culturelle est révolu depuis bien longtemps " annonce Terry Eagleton, un brin nostalgique, au début de son livre After Theory ${ }^{21}$. Quoique multiples, les théories littéraires et culturelles sont assez communément désignées dans la recherche anglo-saxonne par le terme hypostatique «Théorie», écrit au singulier et avec un «T » majuscule. Cette convention renvoie, bien entendu, à une réalité institutionnelle plutôt qu'intellectuelle. La Théorie, précise David Scott Kastan dans son livre Shakespeare After Theory, est évidemment plurielle :

Theory is actually always theories, a heterogeneous collection of paradigms and interests that more or less comfortably cohabit under the same institutional roof but more often than not are intellectually contradictory and incompatible ${ }^{22}$.

Si tous ne partagent pas la nostalgie d'une époque qu'Eagleton qualifie de " grande époque de la Théorie » (" high theory $\left.{ }^{23} »\right)$, chacun s'accorde sur le fait que nous sommes entrés dans une ère post-théorique. Ce qui, à première vue, a de quoi surprendre : est-ce à dire que la longue période de théorisation littéraire, qui a débuté il y a plus de deux mille ans avec Platon et Aristote, est terminée? Comment entendre ce dernier post, qui semble englober tous les posts des théories structuralistes, modernistes, marxistes, féministes, colonialistes et tant d'autres qui se sont développées pendant les dernières décennies? Dans la mesure où l'œil du lecteur n'est pas plus innocent que celui du peintre ou du spectateur d'un tableau, aucun « retour à un état d'innocence pré-théorique n'est évidemment possible, au grand dam des opposants de la Théorie ${ }^{24} \gg$. La lecture est toujours guidée par un «projet anticipant» ou un « horizon d'attente », pour reprendre les expressions de Hans-Georg Gadamer ${ }^{25}$, ceux-ci étant depuis toujours infléchis par la critique littéraire.

21. Terry Eagleton, After Theory [2003], London, Penguin Books, 2004, p. 1. Voir également Martin McQuillan, Graeme Macdonald, Robin Purves et Stephen Thomson (eds.), Post-Theory : New Directions in Criticism, Edinburgh, Edinburgh University Press, 1995, où les éditeurs notent avec humour qu'ils pensent être les premiers à « demander qu'on cesse d'annoncer la fin de l'annonce de la fin de la Théorie » (p. ix).

22. «La Théorie est toujours un ensemble de théories, une collection hétérogène de paradigmes et d'intérêts qui cohabitent plus ou moins confortablement au sein de l'institution mais qui sont souvent contradictoires et incompatibles » (David Scott Kastan, Shakespeare After Theory, London, Routledge, 1999, p. 26).

23. Eagleton, op. cit., p. 2. Pour une critique soutenue de ce qu'il appelle les « excès" voire les « abus » de la Théorie, voir Valentine Cunningham, Reading after Theory, Oxford, Blackwell, 2002.

24. Valentine Cunningham, op. cit., p. 35 ; Terry Eagleton, op. cit, p. 1. Pour une discussion de «l'œil, de l'oreille et de l'esprit innocents », voir E. H. Gombrich, Art and Illusion : A Study in the Pyschology of Pictorial Representation, Oxford, Phaidon Press, 1956.

25. Hans-Georg Gadamer, Vérité et Méthode [1960], Paris, Seuil, 1976 (1960 pour la première publication allemande), p. 104f. 
Le foisonnement sans précédent des méthodologies nouvelles au cours des dernières décennies a très profondément modifié les horizons d'attente des lecteurs et des spectateurs de Shakespeare; il serait vain d'offrir, même sous forme succincte, une synthèse des multiples aspects de la modernité shakespearienne mis en lumière par ces diverses approches théoriques. Même si la "grande époque» de la Théorie est révolue, les approches théoriques sont en pleine évolution. Parmi ces dernières, on notera le présentisme analysé par Jean-Christophe Mayer dans ce volume, ainsi que celle que la critique anglo-saxonne appelle la « Nouvelle esthétique » («New Aestheticism»). Cette dernière nous semble particulièrement intéressante. En effet, pendant cette « période hautement théorique », dans la terminologie de Eagleton, l'étude de l'esthétique du texte littéraire a été marginalisée, voire déconsidérée, face à la critique dite "d'idéologies ». Comme l'écrivent John Joughin et Simon Malpas :

The very notion of the "aesthetic" could be said to have fallen victim to the success of recent developments within literary theory. [...] The rise of critical theory in disciplines across the humanities during the 1980s and 1990s has all but swept aesthetics from the map ${ }^{26}$.

Cette suspicion à l'égard de l'esthétique, nettement plus prononcée dans la critique anglo-saxonne que dans son homologue française, s'explique par la récusation des paradigmes esthétiques autrefois si dominants dans la Nouvelle Critique, à laquelle les théoriciens français étaient peu sensibles. Si les critiques qui plaident pour une nouvelle théorisation de l'esthétique reconnaissent que notre pratique de la lecture est infiniment plus riche, plus « plaisante » et mieux informée par les perspectives ouvertes par la multiplicité des approches critiques développées pendant les dernières décennies, tous constatent que la singularité - la «littérarité 》- du texte littéraire s'en est souvent trouvée négligée voire effacée. L'analyse esthétique qu'un nombre croissant de critiques littéraires et philosophiques appelle de leurs vœux serait, à l'opposé de celle de la Nouvelle Critique ${ }^{27}$, profondément engagée dans les débats politiques et culturels modernes. Comme l'écrivent Joughin et Malpas :

26. John J. Joughin et Simon Malpas, The New Aestheticism, Manchester \& New York, Manchester University Press, 2003, p. 1. «On pourrait affirmer que la notion même de 1'"esthétique" est victime du succès que rencontrent les évolutions récentes de la théorie littéraire [...]. L'émergence de la théorie critique, au cours des années 1980 mais aussi 1990, dans les disciplines qui balisent le champ des sciences humaines, a pratiquement eclipsé l'intérêt pour l'esthétique. »

27. Pour la Nouvelle Critique, le texte littéraire était un objet autonome, quasiment intemporel, véritable " icône verbale », selon le titre d'une étude fondatrice de W. K. Wimsatt, Jr., The Verbal Icon : Studies in the Meaning of Poetry, Lexington, University of Kentucky Press, 1954. 
$[\mathrm{A}] \mathrm{n}$ adequate thinking of modernity requires an investigation of the aesthetic and, reciprocally, the discussion of the impact of art and literature on contemporary culture needs a way of situating this culture in relation to the history of modern politics and philosophy ${ }^{28}$.

Récusant «l'anti-esthétisme» des écrits théoriques des années 1980 et 1990, Isobel Armstrong se propose quant à elle, de forger les éléments d'un nouveau discours esthétique en prise sur les enjeux de la modernité ${ }^{29}$. Elle accorde une importance toute particulière au jeu : " It is in play that it is possible to make an essential cognitive leap which radically changes one's relation to reality ${ }^{30}$. » La réflexion sur le jeu (« the purpose of playing $\left.{ }^{31} »\right)$, qui parcourt toute l'œuvre shakespearienne, souligne, comme le jeu d'enfance dont parle Armstrong, la "potentialité pure » du jeu théâtral, sa capacité de transformer notre perception des choses. Dans le jeu, observe Armstrong, les choses perdent leur force déterminante : ainsi, dans l'imagination de l'enfant, un simple bâton peut devenir un cheval ${ }^{32}$. La manière dont cette " perte » est mise en avant dans le théâtre shakespearien est un des aspects les plus saisissants de sa modernité. C'est d'ailleurs la nature paradoxale de cette "perte» que Keats trouve si éblouissante dans l'œuvre shakespearienne et qu'il décrit par l'expression restée célèbre « negative capability ». Il définit cette " capacité négative» comme « the ability to remain in doubt and uncertainty without any irritable reaching after fact and reason ${ }^{33} »$.

Publié en l'an 2000, The Radical Aesthetic semble annoncer une nouvelle ère, plus propice au développement d'une critique esthétique engagée. En effet, la première décennie du nouveau millénaire a vu la publication de plusieurs essais portant sur l'esthétique et la modernité shakespeariennes, dont Shakespeare and Impure Aesthetics de Hugh Grady ${ }^{34}$. L'auteur, qui depuis une vingtaine d'années a consacré plusieurs ouvrages à la problématique de la modernité shakespearienne, avait déjà esquissé quelques éléments d'une critique esthétique dans The

28. John J. Joughin et Simon Malpas, op. cit., p. 9. «Une pensée pertinente de la modernité requiert une exploration de la sphère de l'esthétique et réciproquement, pour étudier l'impact de l'art et de la littérature sur la culture contemporaine, il convient de se doter d'un moyen de situer cette culture, de la mettre en relation avec l'histoire de la politique et de la philosophie modernes. »

29. Isobel Armstrong, The Radical Aesthetic, Oxford, Blackwell, 2000, p. 1.

30. Ibid., p. 37. « C'est dans le jeu [d'enfance], qu'il est possible d'effectuer ce saut cognitif dont la radicalité permet de bouleverser notre relation à la réalité. »

31. Hamlet, III.ii.20. « [L]e but du jeu théâtral ».

32. Isobel Armstrong, op. cit.

33. Lettres à ses frères, George et Tom Keats, 21 et 27 décembre 1817. « [L]a capacité de demeurer dans le doute et l'incertitude, libéré de la fâcheuse tendance à vouloir établir les faits et à déterminer les raisons. "

34. Hugh Grady, Shakespeare and Impure Aesthetics, Cambridge, Cambridge University Press, 2009. 
Modernist Shakespeare ${ }^{35}$. Cependant, ce n'est que dans notre "présent postmoderniste » et grâce aux nouvelles méthodologies critiques, que nous pouvons re-penser la dimension esthétique de l'œuvre d'art :

A new thinking of the aesthetic has become possible only in our postmodernist present, when the new critical methodologies of our times have permitted us to think of the art-work as disunified, as constituted by internal clashes of discourse and by the insubordination of suppressed materials ${ }^{36}$.

Pratiquement tous les protagonistes shakespeariens sont pris dans un processus de résistance à une subjectivité stable, centrée et « unifiée ». Dans Shakespeare and Impure Aesthetics, Grady étudie les formes théâtrales que peut prendre cette résistance en montrant que c'est souvent en incarnant divers rôles histrioniques que tant de héros et d'héroïnes jouent et se jouent des complexités identitaires.

\section{Modernités shakespeariennes}

Les essais réunis dans ce numéro d'Itinéraires s'inscrivent dans les nouvelles perspectives ouvertes durant la période d'intense théorisation des dernières décennies. Ils proposent de prolonger les débats engagés par la critique et de participer au renouvellement des approches de la question des modernités shakespeariennes.

Dans " "Still keep / My bosom franchised" : Macbeth et l'espace intérieur », Muriel Cunin poursuit la réflexion qu'elle développe dans sa très belle étude sur Shakespeare et l'architecture dans laquelle elle démontre l'importance du rôle du corps, "l'un des principaux dénominateurs communs entre le théâtre et l'architecture ${ }^{37}$ ». Dans sa contribution à ce volume, elle explore les liens problématiques qui unissent intériorité, espace et théâtralité. Comment peut-on montrer l'intériorité, par essence invisible et inaccessible, sur une scène de théâtre? Le paradoxe, comme le souligne l'auteur, semble être absolu : l'intériorité donnée en spectacle n'invaliderait-elle pas l'authenticité de l'être intérieur? C'est un des enjeux majeurs des pièces shakespeariennes où les rapports entre théâtre et illusion, ombre et substance, sont constamment interrogés. Comme l'écrit Katharine Eisaman Maus :

35. Hugh Grady, The Modernist Shakespeare, Oxford, The Clarendon Press, 1991, p. 204-211.

36. Idem, Shakespeare and Impure Aesthetics, op. cit., p. 3. « Il fallait que nous entrions dans la postmodernité pour qu'une pensée de l'esthétique soit à nouveau possible, alors que les nouvelles méthodologies critiques de notre temps nous permettent de penser l'œuvre d'art en termes de désunion, de discours traversé par des conflits internes, de principe d'insubordination à l'œuvre dans l'élaboration d'un site rassemblant des matériaux refoulés. "

37. Muriel Cunin, Shakespeare et l'architecture: Nouvelles inventions pour bien bâtir et bien jouer, Paris, Honoré Champion, 2008, p. 29. 
Le problème de savoir comment on possède un intérieur n'est pas simplement synonyme du problème de savoir comment on habite notre chair; les enjeux semblent être plutôt noués, différenciés de multiples façons même en étant entrelacés et entremêlés ${ }^{38}$.

C'est précisément sur la relation entre corps et esprit-entre le physiologique et le psychologique d'une part et entre le corps et l'espace architectural de l'autre - que Muriel Cunin se penche dans son étude des figures de l'espace intérieur de Macbeth. C'est Macbeth lui-même, tel l'architecte de son propre destin, qui à maintes reprises souligne sa volonté, et sa crainte, de lier dessein interne (disegno interno) et réalisation externe (disegno eterno $)^{39}$. La tension aiguë entre l'être extérieur et l'être intérieur, qui s'installe dès la première rencontre entre Macbeth et les Sorcières et qui se développe à travers le motif du corps/château, est aggravée par le lien somatique qui régit les rapports entre corps et esprit au Moyen Âge, puis à la Renaissance. Les pièces shakespeariennes sont pénétrées par cet imaginaire physiologique qui fait des entrailles le siège des passions. Partant de cette conception d'un corps matériel littéralement chargé de sentiments et de subjectivité, Muriel Cunin démontre comment Shakespeare donne à voir et à entendre l'intériorité de Macbeth et de son épouse. Si l'espace intérieur des assassins s'exprime au travers d'un langage corporel d'une violence inouie, il s'inscrit également dans une série de lieux architecturaux: château, temple, chambres, portes et closet, qui seront tour à tour souillés, fermés comme les corps et les esprits du couple régicide. Ce processus d'intériorisation du Mal, qui s'accompagne de la peur de soi, écrit l'auteur, fonde l'être moderne occidental.

Les trois essais qui suivent s'intéressent, avec des points de vue fort divers, à la question du genre : Gilbert Pham-Thanh étudie Le Portrait de $M r . W . H$. d'Oscar Wilde, qui serait le seul vrai texte critique littéraire des Sonnets shakespeariens aux yeux de Joel Fineman ${ }^{40}$; Eithne O’Neill se penche sur le trope de la femme sacrifiée pour raison d'État, dans deux films : Hamlet de Svend Gard (1920) et Titus Andronicus de Julie Taymor (1994); enfin Agnès Lafont explore la problématique des beautés masculines ambiguës dans le poème narratif Vénus et Adonis (1594), dans Le Marchand de Venise (1596) ainsi que dans Le Songe d'une nuit d'été (1596). Si la question du genre, notamment celui de la femme est débattue un peu

38. Katarine Eisaman Maus, op. cit., p. 182.

39. Comme en témoignent les violentes torsions syntaxiques du soliloque de Macbeth contemplant le meurtre de Duncan : « If it were done, when 'tis done, then 'twere well / It were done quickly » («Si c'en était fait quand c'est fait, alors ce serait bien / On l'aurait vite fait »), qui dessine éloquemment un espace d'entre-deux qui est aussi l'espace intérieur d'un être divisé au moment même où le mal entre en lui. I.vii.1-2, trad. française de Jean-Michel Déprats, Euvres Complètes, Paris, Gallimard, coll. «Bibliothèque de la Pléiade », 2002.

40. "Wilde's The Portrait of Mr. W. H. is the only genuine literary criticism that Shakespeare's sonnets have ever received » (Joel Fineman, Shakespeare's Perjured Eye, op. cit., p. 28). 
partout en Europe durant le Moyen Âge et la Renaissance, la controverse concernant le travestissement semble avoir débuté dans les années 1570, au moment où certaines femmes adoptèrent des habits masculins. La plupart des commentaires, tels que celui de Philip Stubbes, Anatomie of Abuses (1583), associent la dénonciation des femmes masculinisées et la condamnation du caractère efféminé des hommes, indiquant, comme l'écrit Linda Woodbridge, que l'enjeu dépassait nettement celui de la mode pour atteindre la question irrésistible: quelle est, au fond, la différence entre l'homme et la femme ${ }^{41}$ ? Pour Stubbes, comme pour William Averell écrivant en 1588, les femmes déguisées en hommes étaient des monstres; Stubbes parle des « Hermaphrodita », Averell des « Androgini, who counterfayting the shape of either kind, are in deede neither ${ }^{42} \gg$. La mode féminine de porter les habits masculins était, comme le démontre Woodbridge, un phénomène récurrent dans l'Angleterre élisabéthaine et jacobéenne. La littérature de l'époque reflète un vif intérêt pour ces femmes aux airs d'hommes (mannish), et pour ces hommes « efféminés ». En s'interrogeant sur la nature des hommes et des femmes, de nombreux textes suggèrent que les rôles sexuels traditionnels étaient en train d'évoluer dans le monde moderne. Le moyen le plus évident pour explorer les enjeux de cette mutation était le déguisement du travesti au théâtre ${ }^{43}$. Pendant les années 1590, Shakespeare, plus que tout autre dramaturge, avait fait du déguisement travesti un élément essentiel de ses intrigues. C'est aussi durant cette décennie qu'il écrit ses sonnets dont beaucoup, et ceci est tout à fait singulier, sont adressés à un jeune homme. L'identité du dédicataire des Sonnets, un certain Mr. W. H., n'a jamais cessé d'intriguer. The Portrait of $M r . W . H$., publié par Oscar Wilde en 1889, marque un moment critique dans l'histoire de la réception des Sonnets : mi-fiction, mi-essai, le Portrait de Wilde intervient de manière tout à fait singulière dans le débat. En effet, par le biais d'une feinte ingénieuse, consistant à mener à son tour une enquête sur l'identité du mystérieux dédicataire des Sonnets, le Portrait fait émerger des questions d'une tout autre envergure que celles vainement posées, tenant à une réalité biographique quelconque. Ce n'est assurément pas Wilde qui contredirait la devise de Touchstone dans Comme il vous Plaira : « The truest poetry is the most feigning ${ }^{44}$. " En effet c'est autour du beau concetto de la contrefaçon qu'il organise son portrait de Mr. W. H.

41. Linda Woodbridge, Women and the English Renaissance : Literature and the Nature of Womenkind, 1540-1620, Brighton, Sussex, The Harvester Press, 1984, p. 141.

42. William Averell, A Mervailous Combat of Contrarieties, STC 981, 1588, Sig. B1v. "Les Androgènes qui, fabriquant la forme d'un autre genre n'appartiennent ni à l'un ni à l'autre ». Voir aussi Stubbes, Anatoiey of Abuses, STC 23376, 1588, Sig.F5. Cité par Linda Woodridge, op. cit., p. 140.

43. Linda Woodbridge, op. cit., p. 153.

44. III.iii.15 : « La poésie la plus véritable est la plus feinte », trad. Jean-Jacques Mayoux, Paris, Aubier, Montaigne, 1956. 
où, pourrait-on dire « there is very little of the sitter and a great deal of the $\operatorname{artist}^{45}$ ». C'est l'art d'un portraitiste qui « fait jouer niveaux de réalité et régimes discursifs divergents » que Gilbert Pham-Thanh étudie dans l'essai qu'il consacre ici au texte jubilatoire de Wilde. "What's in a name? » demande-t-il dès le début de son analyse de «la masculinité à l'épreuve de Shakespeare relu par Wilde». Avec ce clin d'œil à la question posée par Juliet dans le drame shakespearien, revue par l'amant de Wilde, Lord Alfred Douglas (« What's in a love that dare not speak its name? »), l'auteur introduit un texte où la question du nom (mais lequel?), est omniprésente. «C'est la force de la proposition de Wilde » écrit-il, « que de s'emparer de l'image du Barde, véritable institution nationale, afin d'en faire le champion d'une cause contestable au XIX ${ }^{\mathrm{e}}$ siècle ». Cependant Le Portrait de Mr. W. H. n'est guère un simple plaidoyer pour un amour dont on n'ose pas encore prononcer le nom. Savamment tissée dans l'histoire cocasse d'une enquête sur l'identité de leur dédicataire, cette lecture des Sonnets est à la fois une brillante parodie d'une critique «littéraire » où la «Vérité » de l'œuvre d'art se confond avec un simple fait biographique, et une exploration des "modalités d'expression d'une subjectivité masculine ${ }^{46}$ ». Dans une démarche originale, Gilbert Pham-Thanh met en regard la «situation d'énonciation » de The Portrait de Mr. W. H. avec celle des Sonnets, telle que Francis Meres l'a décrite, afin de mieux faire ressortir ce qu'il appelle une «homosocialité » trans-historique. Derrière l'enquête sur l'identité du bien-aimé des Sonnets, véritable wild goose chase entreprise avec tant d'ardeur par les trois personnages de Wilde, se profile une autre « histoire », celle où l'identité masculine se recompose et où l' « hétéronormativité » de l'idéologie dominante est remise en question. Il s'agit, selon l'auteur, d'une identité masculine inclassable qui « laisse place à une identité queer». Ce queering des identités sexuelles, si palpable dans les pratiques de crossdressing réelles et théâtrales à l'époque élisabéthaine, l'est tout autant, comme le montre cette analyse, dans le texte inclassable de Wilde.

C'est également sur le queering des identités dans deux adaptations cinématographiques de Shakespeare, que Eithne O'Neill se penche : le célèbre Hamlet de Svend Gade, film muet tourné en 1920 avec Asta Nielson dans le rôle d'Hamlet, et le Titus Andronicus de Julie Taymor (1999). L'auteur montre comment ces deux films revisitent la fiction politique médiévale et renaissante des deux corps du roi - corps naturel et corps politique - afin d'explorer le problème des genres (littéraire et sexuel) et de la raison d'État. C'est la manière dont le corps de la femme s'inscrit

45. The Decay of Lying, dans Complete Works of Oscar Wilde, Glasgow, Harper Collins, 1994, p. 1089. " [O]ù le modèle ne figure guère, l'artiste beaucoup. »

46. Voir The Truth of Masks où Wilde observe que «the aesthetic value of Shakespeare's plays does not, in the slightest degree, depend on their facts, but on their Truth » (" La valeur esthétique de l'œuvre shakespearienne ne repose aucunement sur les faits mais sur la Vérité »). Complete Works, p. 1166. 
dans ce paradigme que ces deux adaptations des tragédies de la vengeance mettent en avant, prolongeant ainsi l'interrogation shakespearienne de l'auctoritas du régime patriarcal qui exige que « la femme soit cible et outil de violences ». Gade s'appuie sur le livre d'Edward Vining, The Mystery of Hamlet : An Attempt to Solve an Old Problem (1888), et sur sa théorie « moderne », selon laquelle Hamlet était une femme amoureuse d'Horatio. Mais si Vining pensait résoudre ce qu'il appelle «le vieux problème » d'Hamlet, à savoir le « manque de virilité » mêlé à « la profonde humanité du prince mélancolique », la performance androgyne, sensuelle et ironique d'Asta Nielsen brouille les rapports entre corps biologique et identité sociale, entre le sexe et le genre. De même, selon la lecture que propose ici Eithne O'Neill, le Titus de Taymor explore, à l'instar de la pièce, le glissement des genres, rapprochant la mise à mort de Lavinia, suite au rapt et à l'arrachement de sa langue, au sort d'un Hamlet qui, pour préserver le corps politique, doit taire son identité sexuelle (gender).

L'adaptation cinématographique d'Hamlet avec Asta Nielsen dans le rôle titre est certes une œuvre pionnière, mais la tradition des femmes jouant les rôles masculins shakespeariens au théâtre remonte à la Restauration. Parmi d'autres «Hamlets » on peut citer ceux de Sarah Siddons au XVII et Sarah Bernhardt au XIX ${ }^{\mathrm{e}}$, toutes deux unanimement saluées par le public qui semble avoir trouvé parfaitement normal que le Prince soit joué par des femmes. Marjorie Garber observe qu'au fil du temps, les critiques ont considéré que l'identité de presque tous les caractères shakespeariens, de Richard III à Cléopâtre, sont comme «suspendus entre le masculin et le féminin ${ }^{47}$ ». C'est la perception de cette fluidité ou indétermination de l'identité sexuelle qui a guidé par exemple l'interprétation remarquable de Richard II par Fiona Shaw, dirigée par Deborah Warner en 1995. A propos de cette expérience Shaw a écrit :

I have not made any great conclusions about the event, but I would warn those who wish to try this kind of experiment to make sure they have a spiritual disposition toward the part, to tap the natural androgyne in themselves which will allow the poetic reality to be higher than the representative. [...] The experiments in gender, both socially and artistically, can remind us all of the constant bravery necessary to force the universe of the imagination outwards ${ }^{48}$.

47. Marjorie Garber, Vested Interests : Cross-Dressing and Cultural Anxiety, New York \& London, Routledge, 1991. Cité dans Lizbeth Goodman (ed.), The Routledge Reader in Gender \& Performance, London, Routledge, 1998, p. 180.

48. Foreword, dans Lizbeth Goodman, op. cit., p. xxiv : « Je n'ai pas tiré de grandes conclusions à propos de cette expérience. Mais je conseillerais à celles désireuses de l'expérimenter de s'assurer qu'elles ont une disposition spirituelle envers le rôle en vue de toucher la part d'androgyne en elles, permettant à la réalité poétique de dépasser celle de la représentation. » 
Ce sont également les inscriptions d'un désir renaissant « tout en tension dans lequel rien n'est jamais figé », que déchiffre Agnès Lafont dans l'étude qu'elle consacre ici à « Shakespeare et les amours de Diane : Endymion, Hippolyte et Adonis ». L'auteur montre, à travers 1'exploration d'une nouvelle « constellation » (cluster), qui allie Diane-Lune à Adonis et Diane-Lune à Endymion, comment Shakespeare se réapproprie la tradition mythologique de la déesse lunaire et chasseresse. Les réécritures poétiques et théâtrales que propose Shakespeare de la figure du beau et vertueux jeune homme, célèbrent une beauté masculine ambiguë et désirable, très appréciée du public élisabéthain. Prolongeant les analyses qu'elle fait de ce parangon dans Vénus et Adonis et dans Le Marchand de Venise, Agnès Lafont propose une relecture intéressante de l'histoire du «changeling boy » dans Le Songe d'une nuit d'été, qui fait ressortir toute la puissance de ce que Pierre Brunel appelle l' « irradiation souterraine » du mythe.

Comment la nouveauté était-elle entendue au XVI ${ }^{\mathrm{e}}$ siècle, notamment dans le théâtre shakespearien? Quels étaient ses rapports avec la modernité et le «présentisme »? Ce sont les questions que Jean-Christophe Mayer explore dans son article «Modernité, présentisme et représentation de l'histoire dans le théâtre shakespearien ». Soulignant le caractère paradoxal de la notion de modernité - chaque époque ayant sa propre conception $\mathrm{du}$ «moderne » - l'auteur examine la manière dont la dialectique de l'antique et du nouveau s'inscrit dans les drames historiques, ainsi que dans d'autres types de textes tels que pamphlets, ballades, chroniques, lettres de nouvelles... afin de montrer la complexité des rapports que les Élisabéthains entretenaient avec le passé. Les pièces historiques de Shakespeare ouvraient un espace nouveau, propice à la réflexion sur le passé national, que le théâtre rendait, justement, présent. De nombreux éléments semblent rapprocher présentisme et modernisme. Pourtant, comme le démontre Jean-Christophe Mayer, très critique à l'égard du mouvement « présentiste » contemporain, l'intérêt pour le moderne, pour le présent ou pour l'actuel dont témoignent de nombreux auteurs renaissants, est bien distinct du présentisme prôné par une partie non-négligeable de la critique shakespearienne actuelle.

Les deux articles qui suivent portent sur un des aspects clés de la problématique de la modernité shakespearienne: la traduction. Sans le renouvellement constant des traductions, la modernité de l'œuvre shakespearienne serait infinimentmoins lisible pour ses lecteurs et spectateurs non-anglophones, de même que les adaptations de Shakespeare en langue étrangère resteraient inconnues du public anglophone. Étonnament, la pièce d'Aimé Césaire, Une tempête, parmi les plus célèbres adaptations de La Tempête, n'a jamais été présentée en Angleterre avant qu'elle ne soit donnée à Londres en 1998 dans une nouvelle traduction de Philip Crispin.

Selon Philippe Sollers : 
Traduire Shakespeare est une épreuve épuisante. On allonge, on retarde, on est moins directe, moins cru. On passe moins vite du tragique au comique, et du tragi-comique au lyrisme pur. Moins vite aussi du sarcasme à l'enchantement, de la bestialité à la douceur. Un seul exemple : un personnage fait l'éloge d'un autre personnage, et cela paraît excessif à son interlocuteur. Ce dernier lui dit donc : "You speak him far ». Comment traduire? « Vous allez loin dans le louange »? C'est en effet le sens. Mais la flèche dit : « Vous le parlez loin ». On entend l'anglais, le français l'explique ${ }^{49}$.

Est évoqué ici un certain nombre des questions qui nourrissent la réflexion des traducteurs français. Une seule solution : les éditions bilingues. Reste cependant la question du choix de la traduction. Dans « Modernités shakespeariennes : le cas de la traduction », Marie-Nadia Karsky propose d'explorer la manière dont les traductions françaises contemporaines inscrivent l'œuvre shakespearienne dans la modernité. "Font-elles systématiquement de Shakespeare "notre contemporain", et quels sont les choix de traduction qui en découlent? » Confrontant le travail de quatre traducteurs aux approches distinctes, cette étude fait ressortir la complexité des enjeux de la traduction ainsi que la richesse et la diversité des pratiques de la traduction en France. Il s'agit non seulement de comparer et de discuter des effets de sens qui découlent des choix lexicaux, syntaxiques ou métriques mais aussi, et c'est un des enjeux majeurs des trente dernières années, d'écrire pour le corps et la voix des acteurs.

C'est également de la traduction mais, surtout, d'une réécriture théâtrale célèbre que traite Philip Crispin dans le premier des deux essais sur $L a$ Tempête qui concluent ce recueil. Conseiller littéraire pour le Gate Theatre à Londres durant la saison 1998, et traducteur d'Une tempête d'Aimé Césaire, Philip Crispin s'intéresse ici à la problématique du pouvoir colonial que de très nombreuses relectures de La Tempête ont mises en lumière. En effet, plus que tout autre drame shakespearien, La Tempête, une des dernières pièces de Shakespeare, a inspiré maintes réécritures romanesques, poétiques et théâtrales lors des cinquante dernières années. L'une des premières, celle d'Aimé Césaire, se distingue tant par la beauté de sa langue que par l'acuité de son regard sur les tromperies de la situation postcoloniale dans les anciennes colonies. "Nous vivons», a écrit Malcolm Bowie dans sa critique de la première au Gate Theatre,

dans un âge de brillants Tempêtes... Cependant cette Tempête, du grand poète et politicien martiniquais, Aimé Césaire, n'est pas simplement une nouvelle lecture de Shakespeare mais une pièce originale d'une puissance étonnante ${ }^{50}$.

49. Philppe Sollers commente ici la phrase prononcée par le Second Gentilhomme dans Cymbeline, I.i.24. Discours Parfait, Paris, Gallimard, 2010, p. 111.

50. «We live in an age of brilliant Tempests... yet this Tempest, from the great Martinican poet and politician Aimé Césaire, is not simply a new reading of Shakespeare but an original play of astonishing power » (Malcolm Bowie, « Island Infamy », The Times Literary Supplement, 9 octobre 1998, p. 22). 
L'analyse critique que propose ici Philip Crispin, nourrie d'une part de son expérience théâtrale dans la mise en scène d'Une tempête au Gate Theatre, d'autre part de son travail de traducteur, fait ressortir la puissante modernité ainsi que l'envergure de l'adaptation de Césaire. Une tempête n'est pas simplement une pièce engagée, une réponse ou « writing back» au texte shakespearien : elle revisite la question centrale du pouvoir mais explore également la thématique du rêve et de la musique, en les réinscrivant dans une perspective postcoloniale qui conserve, mais reconfigure sa force transformative. Il s'agit d'une relecture fine et ironique de La Tempête, dont l'esprit et le style sont admirablement traduits dans une version anglaise alerte et souple à la fois.

Le reflet de la modernité que le «miroir » de La Tempête nous tend, écrit Laïla Ghermani, est celui des limites de ce qu'elle appelle, non sans ironie, "the humanist world picture ». Soulignant la grande polyvalence du terme "modernité », l'auteur examine et commente le sens que lui donnent les lectures postcoloniales de la pièce, qui ont largement dominé la critique shakespearienne des années 1980 et 1990. Pour Laïla Ghermani, le problème de la langue est effectivement, comme le souligne l'approche postcoloniale, un des enjeux majeurs de La Tempête. Cependant, l'interprétation proposée ici va à l'encontre de celle-ci et vise à montrer que la modernité shakespearienne s'inscrit dans un questionnement du savoir et de la rhétorique humaniste provoqué par la rencontre avec le nouveau monde. Ébranlant les certitudes des naufragés de l'ancien, cette interrogation laisse apercevoir une autre approche, plus poétique, au monde, ainsi que d'autres savoirs, moins livresques que « sonores et visuels ».

Les approches contrastées d'une des dernières pièces de Shakespeare proposées par Philip Crispin et Laïla Ghermani offrent à ce recueil une conclusion appropriée, en ce qu'elles reflètent la pluralité des perspectives auxquelles se prête l'œuvre shakespearienne et, partant, l'un des aspects les plus saisissants de sa modernité.

Jane Avner Université Paris 13 - PRISMES/PEARL (EA 3977) 